\title{
Characterization and properties of natural fiber polymer composites: a comprehensive review
}

\begin{abstract}
The world is in need of more eco-friendly material, therefore researchers around the globe focus on developing new materials that would improve the environmental quality of products. This need for new green materials has led to the utilization of composites made from raw natural fibers and polymer matrices, and this has become one of the most widely investigated research topics in recent times. Natural fiber composites are an alternative for replacing environmentally harmful synthetic materials and help control pollution problems. In addition, they are low cost, have better mechanical properties and require low production energy consumption. Also, using such materials in construction works, it is possible to improve the sustainability by eliminating construction wastes. Keeping in view all the benefits of natural fiber reinforced polymer composites, this paper first discusses various fabrication techniques employed for the production of these composites and then presents a detailed review of the research devoted to the analysis of their structure and properties by a variety of characterization techniques.
\end{abstract}

Keyword: Natural fiber; Properties; XRD; FTIR; SEM 